\title{
Phase-sensitive quantum effects in Andreev conductance of the SNS system of metals with macroscopic phase breaking length
}

\author{
Yu. N. Chiang and O. G. Shevchenko \\ B. Verkin Institute for Low Temperature Physics and Engineering, \\ National Academy of Sciences of Ukraine \\ Kharkov, Ukraine
}

November 19, 2018

\begin{abstract}
The dissipative component of electron transport through the doubly connected SNS Andreev interferometer indium (S) - aluminium $(\mathrm{N})$ - indium (S) has been studied. Within helium temperature range, the conductance of the individual sections of the interferometer exhibits phase-sensitive oscillations of quantum-interference nature. In the non-domain (normal) state of indium narrowing adjacent to NS interface, the nonresonance oscillations have been observed, with the period inversely proportional to the area of the interferometer orifice. In the domain intermediate state of the narrowing, the magneto-temperature resistive oscillations appeared, with the period determined by the coherence length in the magnetic field equal to the critical one. The oscillating component of resonance form has been observed in the conductance of the macroscopic $\mathrm{N}$-aluminium part of the system. The phase of the oscillations appears to be shifted by $\pi$ compared to that of nonresonance oscillations. We offer an explanation in terms of the contribution into Josephson current from the coherent quasiparticles with energies of order of the Thouless energy. The behavior of dissipative transport with temperature has been studied in a clean normal metal in the vicinity of a single point NS contact.
\end{abstract}

\section{Introduction}

In our previous experiments [1-4] on $S N S$ structures based on clean metals, it has been established that at not too low helium temperatures, the dependence of normal conductance on coherent phase difference between superconducting banks can be preserved even in case the separation $L$ between the $N S$ interfaces exceeds the dimension $L \approx 1 \mu \mathrm{m}$ of the normal layers in $S N S$ disordered nanostructures by three orders of magnitude. It is in those nanostructures that quantum-interference phenomena in dissipative transport have first been observed and are being widely explored today [5-13]. This means that the phase-breaking length $L_{\phi}^{p}$ in clean metals exceeds that length in nanostructures, $L_{\phi}^{d} \sim 1 \mu \mathrm{m}$, by no less than the same value. Low $L_{\phi}^{d}$ in nanostructures seems to be closely related to the short elastic scattering length for electrons: $l_{e l} \sim 0.01 \mu \mathrm{m}$ in 3D-structures and $l_{e l} \lesssim 1 \mu \mathrm{m}$ in 2D-electron gas. The above magnitudes of $l_{e l}$ in metals are typical of high concentration of lattice defects. Insignificant contribution from inelastic scattering at the objects of that kind is evidently the main factor which constrains $L_{\phi}$. In contrast, the macroscopic value of $L_{\phi}$ in clean metals enabled us to extend the spatial range for examining the phase-coherent phenomena and to succeed in observing for the first time the long-range phase coherence at the ratios $L / \xi_{T}>1$, up to $10^{2}$.

Moreover, note that the coherence length $\xi_{T}^{d}=\sqrt{\hbar D / k_{\mathrm{B}} T}$ ( $D$ is the diffusion constant) in dirty (diffusive) limit is expressible in terms of that length $\xi_{T}^{p} \equiv \xi_{T}^{b a l}=\hbar v_{\mathrm{F}} / k_{\mathrm{B}} T$ in clean limit as follows

$$
\xi_{T}^{d}=\left[(1 / 3) l_{e l} \xi_{T}^{b a l}\right]^{1 / 2}, \quad l_{e l} \ll \xi_{T}^{b a l} .
$$


the values $L / \xi_{T}$ are the same, do not coincide but must be related by the equation

$$
\frac{\left(T^{p}\right)^{2}}{T^{d}}=3 \frac{\hbar v_{\mathrm{F}}}{k_{\mathrm{B}} l_{\text {el }}^{d}}\left(\frac{L^{d}}{L^{p}}\right)\left[\frac{\left(L / \xi_{T}\right)^{p}}{\left(L / \xi_{T}\right)^{d}}\right]^{2} .
$$

(We imply that the phase-breaking length $L_{\phi}$ is no less than both $L^{p}$ and $L^{d}$, the separation between $N S$ interfaces in clean and dirty samples, respectively). It thus follows from Eq. (1) that the values of the parameter $L / \xi_{T}=\sqrt{T / E_{c}}$ ( $E_{c}$ is the gap in the density of states [14]) common to both limits at which phase-coherent phenomena behave similarly must be realized at different temperatures. For clean metals, these would be significantly higher. For example, for $L^{p} / L^{d} \sim 10$, the parameter $L / \xi_{T}$ in a clean sample with $l_{e l} \gg 1 \mu \mathrm{m}$ at $T=2 \mathrm{~K}$ is of the same order of magnitude as that in a diffusive sample with $l_{e l} \sim 0.01 \mu \mathrm{m}$ at $T \lesssim 0.1 \mathrm{~K}$. Below we will show that the relationship between the temperature regions within which phase-coherent effects behave analogously in 2DEG-samples with $l_{e l} \sim 1 \mu \mathrm{m}[11]$ and 3D-samples with $l_{e l} \sim 0.01 \mu \mathrm{m}$ [5-7], provided $L^{3 D} / L^{2 D} \sim 1$, is also given by Eq. (1).

The initiation of the phase-coherent phenomena at $L / \xi_{T}>1$ means that long-range phase coherence exists under exponentially small proximity effect for the main group of quasiparticles with the energies $\epsilon \sim T$. In ultra-clean structures those phenomena can therefore be observed within a macroscopic scale and at not too low helium temperatures. This circumstance may appear to be urgent when solving the problem of extracting certain quantum information from various quantum systems through macroscopic channels.

The first evidence for the long-range influence of a superconductor on the conductivity of a normal metal adjacent to it is directly contained in the experimental observables from the structures with a single $N S$ boundary $[15,16]$. The effect was reported to extend over length scales up to $L / \xi_{T} \sim 5 \div 10$ away from the boundary. Subsequently, the interference effects have been discovered in doubly connected $S N S$ systems made of disordered metals (nanostructures) with short $L_{\phi}[5-7]$. Up to now, the variety of $L / \xi_{T}$ studied did not exceed in magnitude the abovementioned. However, as our experiments show, the manifestation of the phase-coherent phenomena in doubly connected $S N S$ systems is not restricted by that interval of $L / \xi_{T}$. Besides, available findings concerning the behavior of those phenomena within the limits of that interval are sometimes interpreted ambiguously (see Fig. 6). Thus, further investigations of the phenomena of such kind are needed.

Below we report on the investigations of the temperature and phase-sensitive features of the conductance of the $S N S$ system in a geometry of an Andreev interferometer. The system was formed by two clean metals in contact, aluminium (in the normal state) and indium, both with $l_{e l} \approx 100 \mu \mathrm{m}$. This allows us to achieve the conditions $L, l_{e l} \gg \xi_{T}=\xi_{T}^{p}$. The ratio $L / \xi_{T}$ was about $10^{2}$. The contribution from supercurrent due to the main group of carriers with the energy $\epsilon \sim T$ was entirely eliminated since all three dimensions of the normal layer in the $S N S$ system exceeded inherent microscopic spatial parameters which are responsible for the proximity effect.

\section{Experiment}

Shown in Fig. 1 is a schematic picture of the doubly connected system investigated made of two metals in contact, aluminium and indium; Inset is an equivalent measuring scheme. Once indium becomes superconducting, the system acquires the $S N S$ configuration of Andreev-interferometer type with an orifice formed by an aluminium bar (of cross section $2 \times 2 \mathrm{~mm}$ ) and an indium strip soldered to each other at the points $a$ and $b$. The orifice area comprises $A=a b \times h \approx 3 \mathrm{~mm} \times 15 \mu \mathrm{m}$.

In our early experiments involving the $S N S$ systems with copper [3, 4], the wide soldered $N S$ contacts of characteristic size $\bar{m}$ could not considerably increase the contact resistance $R_{\text {cont }}$ since $\bar{m} \gg l_{e l}$. In contrast, here, current flows through the narrow contacts $a$ and $b$ with significant spreaded resistance $R_{\text {Sh }}$ of "Sharvin type" which usually develops as $\bar{m} \ll l_{e l}[17]$. The contacts of such size appear in spot soldering indium to aluminium we use in the present work. Note that as we confirmed repeatedly before, the immediate soldering of the metals of the highest purity, with Residual Resistance Ratio at $300 \mathrm{~K}$ and $4.2 \mathrm{~K} R R R \gtrsim 10^{4}\left(l_{e l} \approx 100 \mu \mathrm{m}\right)$, results in the contact barrier height $z$ close to zero, the corresponding transparency coefficient being $t=\left(1+z^{2}\right)^{-1} \approx 1[18]$. $(z \neq 0$ for processing that do not destruct, fully or partially, an oxide layer or other contaminations at the metallic surface). 
total current $I_{N N}$ through a contact between two normal metals must be related to the contact area $A_{\text {cont }}$ by the equation [18]

$$
I_{N N}=2 \nu(\epsilon) e^{2} v_{\mathrm{F}} A_{\text {cont }} U_{\mathrm{Sh}} t \equiv U_{\mathrm{Sh}} / R_{\mathrm{Sh}}
$$

in which $\nu(\epsilon)$ is the density of states in either contacted metal while $U_{\mathrm{Sh}}$ is the voltage drop at the spreaded resistance $R_{\mathrm{Sh}}$. Selecting aluminium, with normal conductivity $\sigma_{N}=(1 / 3) e^{2} v_{\mathrm{F}} \nu(\epsilon) l_{e l}$, as a 3D-part of the system, from Eq. (2) we get the contact area $A_{\text {cont }}$

$$
A_{\text {cont }}=(1 / 6)\left(l_{e l}^{\mathrm{Al}} / L^{\mathrm{Al}}\right)\left(U^{\mathrm{Al}} / U_{\mathrm{Sh}}\right) A^{\mathrm{Al}} .
$$

Here, we took into consideration that $I_{N N}=j^{\mathrm{Al}} A^{\mathrm{Al}}=j_{\text {cont }} A_{\text {cont }}\left(j^{\mathrm{Al}}\right.$ and $j_{\text {cont }}$ are the current densities in aluminium and in the contact, respectively). In addition, $A^{\mathrm{Al}} \approx 4 \mathrm{~mm}^{2}$ is the cross section area of aluminium bar; $L^{\mathrm{Al}} \approx 1.5 \mathrm{~mm}$ is the length of the corresponding $\mathrm{Al}$ part between one of the contacts, say $a$, and the measuring probe $V_{2}$ (ac in Fig. 1 ); $U^{\mathrm{Al}}=I_{N N} R^{\mathrm{Al}}$ is the voltage across the Al part; $R^{\mathrm{Al}}$ is the resistance of that part measured independently. The potential difference $U_{\mathrm{Sh}}$ across the spreaded resistance can be found from the voltage $U$ measured at the probes $V_{1}$ and $V_{2}$ :

$$
U_{\mathrm{Sh}}=U-I_{N N}\left(R^{\mathrm{Al}}+R_{\text {narr }, N}^{\mathrm{In}}\right)
$$

where $R_{n a r r, N}^{\mathrm{In}}$ is the resistance of the indium narrowing next to the contact (see Inset to Fig. 1) in the normal state. Other quantities necessary for estimating $A_{\text {cont }}$ have been measured to be as follows.

$$
R^{\mathrm{Al}} \approx 4 \cdot 10^{-10} \Omega ; R_{\mathrm{Sh}} \approx 1.1 \cdot 10^{-8} \Omega ; R_{\text {narr }, N}^{\mathrm{In}} \approx 1.7 \cdot 10^{-8} \Omega .
$$

Hence, Eq. (3) yields that the characteristic dimension $\bar{m}$ of the "spot" at the contacts $a$ and $b$ may amount to approximately $25 \mu \mathrm{m}$, this corresponding to inequality $l_{e l}>\bar{m}$, so that an additional spreaded resistance $R_{\text {Sh }}$ may appear. In our experiment, $R_{\text {Sh }}$ exceeds the resistance of the normal region $a c$ by two orders of magnitude.

We performed dc four- and three-terminal measurements using normal (copper) leads $I_{1}, V_{1}$ and $I_{2}, V_{2}$. The former were soldered to indium beyond the narrowings while the latter were spot welded onto aluminium. Measuring current $(I \approx 0.5 \mathrm{~A})$ was inserted into the system via the leads $I_{1}, I_{2}$. Once the $N S$ contacts have been prepared, the indium narrowing next to the contact $b$ was further thinned down (by drawing) to bring the resistances of the interferometer arms $d b f$ and $d a f$ (see Inset to Fig. 1 ) into the relation $R_{d b f} \gg R_{d a f}\left(R_{d b f} \equiv R_{n a r r, b}^{\mathrm{In}} \sim 10^{-3} \Omega\right)$. Assuming this, practically all the current injected was passed through the circuit " $I_{1}-$ indium narrowing - contact $a$ - aluminium $-I_{2}$ ". The macroscopic phase difference was still controllable.

The phase difference was varied by applying external magnetic field $H_{e}$ from the rectangular wire turn carrying the current $I_{H_{e}}$. The turn was attached directly onto the aluminium bar face in such a way that the plane of the interferometer orifice was parallel to that of the turn. This circumstance simplifies calculating the field strength in the orifice region. To compensate the extraneous fields, the sample with the turn was placed inside a closed superconducting screen.

The potential difference between leads $V_{1}, V_{2}$ was measured by the device using a thermomagnetic superconducting modulator [19], with an accuracy to no more than $(0.5 \div 1) \times 10^{-12} \mathrm{~V}$. This allows us to study the effects of the magnitude of down to $0.1 \%$ in the conductance of macroscopic $N$-regions. The error in measuring current and temperature ranges from $0.001 \%$ up to $0.01 \%$. Current-voltage characteristics were verified to be linear over a wide interval of currents.

\section{Results and discussion}

\section{1 $\quad H_{e}=0$. Temperature dependence}

Curves 1 and 2 in Fig. 2 depict the temperature dependence of the potential difference $U$ normalized by the measuring current which is inserted into the system via the leads $I_{1}$ and $I_{2}$, assuming $R_{\text {cont }}^{a} \ll R_{\text {cont }}^{b}$. When the temperature was lowered down to the critical superconducting point for the bulk indium part $T_{c}^{\mathrm{In}}=3.41 \mathrm{~K}$ and the $N S$ boundary developed, a step-like increase in the resistance of eacsection was revealed, of the type we pioneered in observing in 1988 [15]. We believe this to be 
that Andreev reflection can manifest itself macroscopically only at temperatures somewhat below $T_{c}$ for bulk indium when the superconducting energy gap grows in value noticeably. In particular, at $T=3.2 \mathrm{~K}, \Delta(T) \approx 0.1 \Delta(0)$ while $\left[1-T / T_{c}\right] \approx 0.06$. Analyzing the contribution into resistance from individual parts of the $S N S$ system, estimated in Sec. 2, and curves 1 and 2 in Fig. 2, one concludes that only indium narrowing can be responsible for the height of the jump in resistance near $T_{c}^{\mathrm{In}}$ and its further changing with cooling down to $T \approx 1.8 \mathrm{~K}\left(R_{\mathrm{Sh}}\right.$ does not depend on temperature and $\left.R^{\mathrm{Al}} \ll R_{\text {narr }, N}^{\mathrm{In}}\right)$. It is seen that the resistance of the narrowing at $N S$ configuration of the system $\left(\approx 3.4 \times 10^{-8} \Omega\right.$ at $\left.T=3.2 \mathrm{~K}\right)$ is twice as large as that resistance in $N N$ state $\left(\approx 1.7 \times 10^{-8} \Omega\right.$ at $\left.T=3.5 \mathrm{~K}\right)$.

According to the microscopic theory [21, 22] such an increase in the normal resistance assuming Andreev reflection occurs is due to the doubling of the scattering cross section for electrons at the impurities located within the range of order of the coherence length $\xi_{T}^{p}$ away from the $N S$ boundary (for indium, $\xi_{T}^{p} \approx 10 \mu \mathrm{m}$ at $T \approx 3 \mathrm{~K}$ ). This can be detected in case $L \sim \xi_{T}^{p}$ where $L$ is the length of a normal metal layer measured from the boundary. The estimation for the dimensions of the narrowing, with the magnitudes of $\mathrm{RRR}^{\mathrm{In}} \approx 4 \times 10^{4}, A_{\text {cont }}$, and $R_{\text {narr }}^{\mathrm{In}}$, shows that the distance from the "spot" to the bulk indium section of the system where the $N S$ boundary arises at $T<T_{c}^{\mathrm{In}}$, is of order of $10 \mu \mathrm{m}$, i. e., is comparable to $\xi_{T}^{p}$. Therefore, the above theoretical conclusion about the twofold enhancement of the resistance seems to be directly confirmed for the first time. The maximum increase in the resistance we managed to observe before did not exceed $60 \%$ [23].

In Fig. 3 (curves 2-5) we present the conductance measured on the opposite side from the contact $a$, within the normal aluminium part, as a function of the thickness of the normal layer next to $N S$ boundary, i. e., of the separation $L_{N S}$ between the normal lead $N$ and the superconducting point contact $a$. The measurements were performed by dc four-terminal zero method which allowed us to exclude the contribution $R_{\mathrm{Sh}}+R_{n a r r}^{\mathrm{In}}$. In this case, the ring of the interferometer was interrupted. For comparison, in Fig. 3, we also show the temperature-dependent resistance of the same aluminium sample (curve 1) measured using only normal leads.

The curves in Fig. 3 illustrate the evolution of the increase in resistance of the next-to-contact aluminium layer dependent on $L_{N S}$, with the rise of $N S$ boundary. It is seen from comparing Figs. 2 and 3 that the effect of increasing in the normal resistance observed on each side of the contact $a$ is similar to the effects evidenced in other $N S$ systems, with other metals, at arbitrary area of the $N S$ boundaries, and dissimilar arrangements of the leads [2, 23]. The nature of the effect as mentioned above is associated with the interference of the coherent Andreev reflected electrons while its magnitude only depends on the ratios $\xi_{T} / L_{N S}, l_{e l}$ provided $L_{\phi} \gg l_{e l} \gg \xi_{T}$ and $L_{N S}<L_{\phi}$. The results in Fig. 3 thus show once again that the long-range phase coherence in a clean metal at the temperatures investigated can be sustained within macroscopic distances, of no less than $1.5 \mathrm{~mm}$ in our case, at $L / \xi_{T} \approx 10^{2}$. This fact, as well as our previous findings [1-4, 23], points out that the phase breaking length is at least of the same order or greater.

The temperature-dependent resistance of both indium and aluminium measured on each side of the contact $a$ below the jump temperature where $\xi_{T}<L_{N S}$, is governed by the same power law $\sim T^{3.5}$ (see Figs. 2 and 3). In Ref. 2, we detected similar behavior of aluminium comprising a part of $N S$ system when measurements were carried out in a different way. We find this to be an additional confirmation that the temperature-dependent phase-breaking length does determine the temperature dependence of the conductance of a metal layer as a whole, within the range $\xi_{T}<L_{N S}<L_{\phi}$, under multiple Andreev reflections [2].

\section{$3.2 \quad H_{e} \neq 0$}

\subsubsection{Nonresonance oscillations}

The potential difference $U$ measured across the leads $V_{1}, V_{2}$ as a function of the external magnetic field $H_{e}$ at $T=3.2 \mathrm{~K}$ exhibits an oscillating component with the period $(h c / 2 e) / A, A$ being the area of the orifice (see Fig. 1). The amplitude of the oscillations is plotted in Fig. 4, curve 1, in relative units $U / I \propto\left(R_{H}-R_{H=0}\right) / R_{H=0}$. Its absolute value comprises $\Delta(U / I)=\left(R_{\text {max }}-R_{\text {min }}\right) \approx$ $4.5 \times 10^{-10} \Omega$ which corresponds to approximately $2 \%$ of the indium narrowing resistance $R_{\text {narr }, S N}^{\operatorname{In}}$. Here, $R_{\min }$ stands for $R_{H=0}$. In Fig. 5, the temperature dependence of the difference $\Delta(U / I)=$ 
dependence $\mathrm{d}(U / I) / \mathrm{d} T$ in Fig. 2, points out that the domain intermediate state in indium narrowing is realized only after reducing the temperature down to $T \approx 3.1 \mathrm{~K}$. Independent analysis, accounting for the size $L_{\text {narr }}^{I n}$, leads to the same conclusion. Indeed, at $T \gtrsim 3 \mathrm{~K}$, the length $L_{\text {narr }}$ in self-magnetic field $(\sim 10 \mathrm{Oe})$ of measuring current does not satisfy the condition for arising the domain structure with the number of domains greater than 1 [3]. Moreover, once the temperature reduced lower than 3K, magneto-temperature resistive oscillations appear (see Inset to Fig. 5), with the period $\Delta_{H_{c}(T)} \sim$ $h c / e \xi_{H}^{2}$ in critical magnetic field where $\xi_{H} \approx 2 \sqrt{q R_{\mathrm{L}}\left[H_{c}(T)\right]} \sim 1 \mu \mathrm{m}$ at $T=3.0 \mathrm{~K}(q$ is the screening radius for an impurity, $R_{\mathrm{L}}\left(H_{c}\right)$ the Larmour radius) [1,3]. This fact is supposed to result from the transition of the indium narrowing into the domain intermediate state and thus is an additional evidence for that transition occurs at temperatures not higher than $\approx 3.1 \mathrm{~K}$.

Compare the parameters of oscillations observed at $3.2 \mathrm{~K}$ (curve 1 in Fig. 4) with theory and the data thus far available from other investigators. In Fig. 6, we plotted the most characteristic data from Refs. 7, 11, and 13 on the temperature dependences of the relative amplitudes $\left|\Delta R / R_{N}\right|$ of the resistive oscillations as a function of the parameter $T_{\mathrm{Th}} / T \equiv\left(\xi_{T} / L\right)^{2}$, with the "Thouless temperatures" $T_{\text {Th }}$ adopted by the authors. Also, shown is the theoretical curve [24] $\left|\Delta R / R_{N}\right|=$ $\left|R_{\max }-R_{N}\right| / R_{N}$, where $R_{\max }$ and $R_{N}$ are the resistances in maximum and minimum of the oscillations, respectively. The curve has been received by numerical simulation for cases $\phi=\pi$ and $\phi=0$ assuming $T_{\mathrm{Th}}=D / \pi L^{2}$. Apperent discrepancy between the data presented and theory [24], as well as between different experiments, can be almost entirely removed if one takes an energy criterium $T^{*}=D / 2 \pi L^{2}$ received for dirty limit in Ref. 25 as a gap in density of states generated when coherent excitations are localized in a normal part between NS interfaces due to Andreev reflections. The same results as in Fig. 6 are presented in Fig. 7b, using the separation between superconducting "mirrors" as $L$ and the above parameter $T^{*}$ as "Thouless temperature". It can be seen that the experimental oscillation amplitudes modified in such a way follow a certain law in the parameter $T^{*} / T$. This feature results immediately from the theory by Aslamazov, Larkin, and Ovchinnikov [25] developed as early as 1968. In fact, consider that quasiparticle dissipative current is a difference between the total current and its non-dissipative part and is proportional to $f(\cos \Delta \chi), \Delta \chi$ being the macroscopic phase difference [26]. Based on the analytical expressions for non-dissipative current $(\sim \sin \Delta \chi)$ from [25] we thus find

$$
\frac{R_{\Delta \chi=\pi / 2}}{R_{N}}=\left[1-(1 / \pi) \frac{L}{\xi_{T}} \exp \left(-\left(\frac{L}{\xi_{T}}+1\right)\right) \ln \left(\alpha\left(\frac{L}{\xi_{T}}\right)^{-2}\right)\right]^{-1} .
$$

Here, $L / \xi_{T} \equiv\left(T / T^{*}\right)^{1 / 2}, \alpha$ is a coefficient of order of unity.

The curve thus calculated for $\alpha=2$ is plotted in Fig. 7a, together with the curve $R_{\Delta \chi=0} / R_{N}$ from Ref. 24. It can easily be seen that both curves predict the existence of long-range phase coherence, i. e., a non-exponential decay of the oscillating dissipative component in the conductance of an $S N S$ system under $L / \xi_{T} \gg 1$. The two curves differ from one another by the factor $\sqrt{2}$ in their position relative to $L / \xi_{T}$ scale to the extent as $\xi_{T}^{[24]}$ differs from $\xi_{T}^{[25]}$. The same theoretical curve for the relative oscillation amplitude $\left|\Delta R / R_{N}\right|$ from Ref. 25 is shown in Fig. 7b by dashed line. It describes properly the position of all the experimental data from Fig. 6 on the temperature scale which fact supports the conclusion about the relationship between the temperature intervals deduced from Eq. (1). (When handling the experimental data from Ref. 11 we took into consideration that the normal-part size in one of the directions exceeded $l_{e l}$ and did not satisfy the ballistic criterion for $\xi_{T}^{p}$. In this case, the Thouless temperature must be estimated in a different way as we have done). Moreover, from the curve [25], we can also obtain correct quantitative estimation for the oscillation amplitude in the corresponding temperature intervals. Excluded are the data reported in Ref. 11 where the total sample resistance is taken as $R_{N}$ rather than the resistance of the section between the "mirrors".

Hence, as the above analysis corroborates, the experimental results $[7,11,13]$ have most likely been received in the range of the parameter $L / \xi_{T}>1\left(T^{*} / T<0.3\right)$, i. e., in "dirty" limit. In contrast, it seems to be reasonable to attribute the oscillations observed from the indium narrowing next to contact $a$ at $T=3.2 \mathrm{~K}$ to the quasiballistic regime $L_{n a r r}^{\mathrm{In}} / \xi_{T} \sim 1$. In this regime, a characteristic temperature must be $T^{b a l}=\hbar v_{\mathrm{F}} / k_{\mathrm{B}} L$. The oscillation amplitude calculated employing this parameter as $T^{*}$ and $L_{n a r r}^{\mathrm{In}}$ as $L$ is shown in Fig. $7 \mathrm{~b}$ as a square. Its location on the temperature scale agrees with theory [25]. 
At $T \sim 2 \mathrm{~K}$, we observe the oscillations in a magnetic field (curve 2 in Fig. 4 ) which have a resonant form unlike those observed at $3.2 \mathrm{~K}$. Their period does not change and is given by $(h c / 2 e) / A$. We assume their nature to be connected with the peculiarities of the phase-coherent interference in aluminium. The reasonings are as follows. First, at $T \sim 2 \mathrm{~K}$, the resistance of indium part becomes as low as that of aluminium between points $a$ and $c$ (see Inset to Fig. 1). Second, the phase of resonant oscillations is shifted by $\pi$ relative to that of nonresonance oscillations. (It is worth noting that the above inversion of the resistive oscillation phase has also been observed in other works, for example, [11, 13] in which the interferometer geometry and measuring technique differ from those in our experiment).

We should emphasize once more that in the system investigated the phase-breaking length is either much greater than the separation between the injectors of electrons, as in case of indium narrowing in the domain state, or of order of that separation, as in case of aluminium part ( $\sim 1 \mathrm{~mm}$ in length). This is the first condition necessary for phase-coherent quasiparticle phenomena to reveal themselves in the conductance of $S N S$ systems with large separation between the interfaces. Next principal consideration which has been discussed in detail in theory [27] is the limitation on the dimensions of injectors which act as reservoirs of quasiparticles. In the ballistic regime, an electron beam must be splitted at the injector site in order that Andreev-reflected excitations, with low energies $\epsilon \leq E_{c} \sim \hbar v_{\mathrm{F}} / L$, follow quasiclassical paths connecting both superconducting "mirrors", instead of returning into the injector after the first reflection. Under such conditions, the coherent phase difference between the "mirrors" can be established. As shown [27], the diameter of the injector-reservoir should not exceed de Broglie wave length $\lambda_{\mathrm{B}}$ (this was first noted in Ref. 28). It is not difficult to understand that this limitation loses its meaning if a superconducting bank serves as at least one of the injectors since in this case the splitting is not needed for initiating a trajectory connecting both banks. In our SNS system, current is introduced through one of the "mirrors" (Fig. 1) so that the above limitation is absent, for both the indium narrowing in the ballistic regime and the aluminium section in the regime close to the diffusive one.

Since the separation between Andreev levels is $\sim \hbar v_{\mathrm{F}} / L$ we can assume that it is the aluminium part in which $2 \mathrm{~K}$-oscillations arise connected with the fine structure of Andreev spectrum for low-energy electrons. As mentioned, the oscillations have a resonant form, in contrast to both $3.2 \mathrm{~K}$-oscillations in indium narrowing and the oscillations we observed before in normal copper part of the $S N S$ system with large area of "mirrors" and current injected not through the "mirrors" [4]. As can be seen from Fig. 4, the amplitude of the resonant oscillations relative to the resistance of aluminium between the "mirrors" is about $4 \%$, in accordance with the ratio $E_{c} L / T$ for aluminium rather than indium narrowing. Theory $[27,29]$ yields that the resonant oscillations can be expected to result from the degeneracy of the transverse modes on the Fermi level. In this case, the Andreev-level energies

$$
\epsilon_{n}^{ \pm}=\frac{\hbar v_{\mathrm{F}}}{2 L}[(2 n+1) \pi \mp \Delta \chi]
$$

( $\Delta \chi$ is the macroscopic coherent phase difference between the "mirrors") go to zero as soon as $\Delta \chi=$ $(2 n+1) \pi$. The degeneration condition therefore assumes that the phase of resonant oscillations should be inversed relative to that of nonresonance oscillations, the latter being given by $\Delta \chi=2 n \pi$. The inversion of this kind we observe for the oscillations of resonant form.

\section{Conclusions}

The phase-coherent component of the dissipative electron transport has been studied in a doubly connected hybrid system formed by clean metals, In and Al, with elastic mean free path about $100 \mu \mathrm{m}$ and phase-breaking length greater than $1 \mathrm{~mm}$. The device has a geometry of an Andreev SNS interferometer. The characteristic dimension of the $N S$ interfaces is less than the mean free path while the size of the normal part between the interfaces is comparable to the macroscopic phase-breaking length. A number of phase-sensitive effects of quantum-interference nature have been revealed in cases when current is injected both through one of the $N S$ interfaces and beyond them. The effects result from the presence of coherent component due to Andreev reflection. We distinguished the effects originating 
interface and normal aluminium.

The resistive oscillations with the period $\Phi_{0} / A$ observed in an external magnetic field at $T=3.2 \mathrm{~K}$ we relate to the behavior of the electron transport in the indium narrowing in the normal (non-domain) state. In the domain intermediate state of the narrowing, the oscillations of the magneto-temperature type have been revealed, their period being $2 \Phi_{0} / \xi_{H_{c}(T)}$.

At $T \lesssim 2 \mathrm{~K}$, the resistive oscillations of resonant form are detected, with the phase shifted by $\pi$ in reference to that of nonresonance oscillations. We suggest that the resonant oscillations are exhibited by macroscopic normal-aluminium section of the system. The oscillations originate from the degeneracy of the transverse Andreev modes for coherent quasiparticles, with energies of order of the Thouless energy, at the Fermi level. For such quasiparticles, the transport regime may be ballistic when moving through a normal region of macroscopic size $L$, between a reservoir and $N S$ interfaces, if $l_{e l} \gg \xi_{T}^{b a l}$. In the ballistic regime, on condition that $N S$ interface serves as one of the electron injectors, the manifestation of the phase coherence does not depend on $L$ as long as $L \leq L_{\phi}$. It can thus be assumed that the observation of the phase-sensitive effects in the conductance of macroscopic $S N S$ systems is only restricted by those values of $L$ at which the normal reflection from $N S$ interfaces becomes dominant. This occurs provided $E_{c} / T<\sqrt{E_{c} / E_{\mathrm{F}}}[27]$ whence it follows that at $T \sim 2 \mathrm{~K}$ and $L_{\phi} \rightarrow \infty L$ must be over $10 \mathrm{~cm}$. This is a limiting value of the distance between interfaces at which the long-range phase coherence can manifest itself at helium temperatures $\left(\left(L / \xi_{T}^{b a l}\right) \sim 10^{4}\right)$.

The phase-sensitive quantum phenomena in the conductance of the $S N S$ system formed by clean metals we observed experimentally at not too low helium temperatures under the condition $\left(L / \xi_{T}^{b a l}\right) \sim$ $10^{2}$ can be reasonably explained in the limits of the above $L$-scale for long-range phase coherence due to the contribution from low-energy coherent excitations with the energy $E_{c} \ll T, \Delta$.

\section{References}

[1] Yu. N. Chiang (Tsyan), Pis'ma Zh. Eksp. Teor. Fiz. 71, 481 (2000) [JETP Lett. 71, 334 (2000)].

[2] Yu. N. Chiang, S. N. Gritsenko, and O. G. Shevchenko, Zh. Eksp. Teor. Fiz. 118, 1426 (2000) [JETP 91, 1235 (2000)].

[3] Yu. N. Chiang and O. G. Shevchenko, Fiz. Nizk. Temp. 27, 1357 (2001) [Low Temp. Phys. 27, $1000(2001)]$.

[4] Yu. N. Chiang and O. G. Shevchenko, Pis'ma Zh. Eksp. Teor. Fiz. 76, 794 (2002) [JETP Lett. 76, 670 (2002)].

[5] V. T. Petrashov, V. N. Antonov, P. Delsing, and T. Claeson, Phys. Rev. Lett. 70, 347 (1993); 74, 5268 (1995).

[6] A. Dimoulas, J. P. Heida, B. J. v. Wees, T. M. Klapwijk, W. v. d. Graaf, and G. Borghs, Phys. Rev. Lett. 74, 602 (1995).

[7] H. Courtois, Ph. Gandit, D. Mailly, and B. Pannetier, Phys. Rev. Lett. 76, 130 (1996).

[8] P. Charlat, H. Courtois, Ph. Gandit, D. Mailly, A. F. Volkov, and B. Pannetier, Phys. Rev. Lett. 77,4950 (1996).

[9] H. Takayanagi and T. Akazaki, Phys. Rev. B 52, R8633 (1995); Physica B 249-251, 462 (1998).

[10] S. G. den Hartog, B. J. van Wees, Yu. V. Nazarov, T. M. Klapwijk, and G. Borghs, Physica B 249-251, 467 (1998).

[11] E. Toyoda and H. Takayanagi, Physica B 249-251, 472 (1998).

[12] P. G. N. de Vegvar, T. A. Fulton, W. H. Mallison, and R. E. Miller, Phys. Rev. Lett 73, 1416 (1994). 
P. Delsing, and T. Claeson, Phys. Rev. B 60,14589 (1999).

[14] F. Zhou, P. Charlat, B. Spivak, and B. Pannetier, J. Low Temp. Phys. 110, 841 (1998).

[15] Yu. N. Chiang (Tszyan) and O. G. Shevchenko, Fiz. Nizk. Temp. 14, 543 (1988) [Sov. J. Low Temp. Phys. 14, 299 (1988)].

[16] V. T. Petrashov, V. N. Antonov, S. V. Maksimov, and R. Sh. Shaikhaidarov, Pis'ma Zh. Eksp. Teor. Fiz. 58, 48 (1993) [JETP Lett. 58, 49 (1993)].

[17] Yu. V. Sharvin, Zh. Eksp. Teor. Fiz. 48, 984 (1965).

[18] G. E. Blonder, M. Tinkham, and T. M. Klapwijk, Phys. Rev. B 25, 4515 (1982).

[19] Yu. N. Chiang, Prib. Tekhn. Eksp. 1, 202 (1981).

[20] A. F. Andreev, Zh. Eksp. Teor. Fiz. 46, 1823 (1964) [Sov. Phys. - JETP 19, 1228 (1964)]; 49, 655 (1965) [22, $455(1965)]$.

[21] J. Herath and D. Rainer, Physica C 161, 209 (1989).

[22] A. M. Kadigrobov, Fiz. Nizk. Temp. 19, 943 (1993) [Low Temp. Phys. 19, 670 (1993)]; A. M. Kadigrobov, R. I. Shekhter, and M. Jonson, Physica B: Condens. Matter 218, 134 (1996).

[23] Yu. N. Chiang and O. G. Shevchenko, Fiz. Nizk. Temp. 25, 432 (1999) [Low Temp. Phys. 25, 314 (1999)].

[24] Yu. V. Nazarov and T. H. Stoof, Phys. Rev. Lett. 76, 823 (1996).

[25] L. G. Aslamazov, A. I. Larkin, and Yu. N. Ovchinnikov, Zh. Eksp. Teor. Fiz. 55, 323 (1968) [Sov. Phys. - JETP 28, (1968)].

[26] A. I. Svidzinskii, Prostranstvenno-neodnorodnye zadachi teorii sverkhprovodimosti, Moscow; Nauka (1982) [Spatial-nonhomogeneous problems in the theory of superconductivity].

[27] H. A. Blom, A. Kadigrobov, A. M. Zagoskin, R. I. Shekhter, and M. Jonson, Phys. Rev. B 57, 9995 (1998-II).

[28] B. Z. Spivak and D. E. Khmel'nitskii, Pis'ma Zh. Eksp. Teor. Fiz. 35, 334 (1982) [JETP Lett. 35, 413 (1982)].

[29] I. O. Kulik, Zh. Eksp. Teor. Fiz. 57, 1745 (1969) [Sov. Phys. - JETP 30, 944 (1970)]. 
Fig. 1. Sketch of the $S N S$ interferometer and equivalent measuring scheme (Inset). Crosshatched is the bulk part of indium.

Fig. 2. Temperature-dependent resistance of indium narrowing in the vicinity of contact $a$ at $R_{\text {cont }}^{a} \ll R_{\text {cont }}^{b}$ (curves 1,2) and its derivative (curve 3). Circles and triangles depict the data from minimum $\left(H_{e}=0\right)$ and maximum $\left(H_{e}=0.3 \mathrm{mOe}\right)$ of the resistive oscillations observed at $T=3.2 \mathrm{~K}$. The jump on the curves 1,2 corresponds to the twofold increase in the resistance of indium narrowing after the initiation of the $N S$ interface (spreaded contact resistance included).

Fig. 3. Effect of increasing in the resistance of aluminium part near the interface (in the region $a c$, see Fig. 1) as a function of the separation between $N$ probe and point $N S$ interface. $H_{e}=0$.

Fig. 4. Phase-sensitive component of the resistance of the interferometer with $R_{a} \ll R_{b}$ vs external magnetic field. Nonresonance oscillations from indium narrowing at $T=3.2 \mathrm{~K}$ (triangles) and resonant oscillations from aluminium at $T=2 \mathrm{~K}$ (circles).

Fig. 5. Temperature dependence of the difference between the resistance of the interferometer at $H_{e}=0.3 \mathrm{mOe}$ and $H_{e}=0$ (Inset is the same enlarged).

Fig. 6. Temperature dependence of the amplitude of phase-sensitive nonresonance conductance oscillations from experiments [7], [11], and [13]. Solid curve is theory [24].

Fig. 7. $a$ - Resistance of an $S N S$ system against the parameter $L / \xi_{\mathrm{T}}$ as follows from [24] and [25]; $b$ - Temperature dependence of the amplitude of nonresonance conductance oscillations from [7], [11], and [13] (see Fig. 7) modified in accordance with the theory [25]. 